\title{
Discussion on Cultivation of Students' Innovative Ability During a Graduation Thesis of an Undergraduate Gan Zhao
}

\author{
College of Life Science , South China Agricultural University , Guangzhou 510642 , China ; \\ E-mail:zg200010@163.com
}

Keywords: Undergraduate; Graduation thesis; Discussing; Cultivation; Students' innovative ability.

\begin{abstract}
On how to cultivate students' innovating ability during a graduation thesis of an undergraduate, it is thought here that consulting and tidying up references to select a proper thesis, protocoling experiments and designing experimental methods scientifically, doing experiments accurately,and summarizing scientifically the experimental results should be pay attention to.

The graduation thesis is the last course and extremely important link of an undergraduate during his or her undergraduate studies. Its importance has long been known and a lot of efforts have been made to improve it in China (1-11).

It was thought that the education on innovation is a kind of education with fostering and developing students' innovative consciousness, innovative spirit and innovative ability as the starting point (1). During the graduation thesis stage, how to use integratedly basic knowledges, theories and basal experimental techniques to select a proper thesis, how to determine experimental schemes, how to carry out experimental processes accurately, how to seek for methods to solve the problems occurring in the experiments, how to analyze scientifically the experimental results and to evaluate their significance, and how to forecast the subsequent researches, all these are the basal trainings for an undergraduate to get. Culturing all the quality will be positive for undergraduates to do scientific researches independently and to approach their life scientifically.
\end{abstract}

\section{Consulting and tidying up references to select a proper thesis}

Consulting and tidying up references are preconditions, otherwhile selecting a proper thesis is the result. Though undergraduates have studied relational basic subjects and professional basic subjects, they often feel very ignorant about their interests, researching field and researching thesis when just getting into the graduation thesis stage. What is more, some students do not think highly of the graduation theses at all(2). At this moment their teachers or advisors will be the linchpin.Here the advisor should guide the undergraduate to consult and to tidy up the references in researching field and to make full use of basic knowledges and theories to seek for the proper thesis to study.

What is a proper thesis ? The most fundamental standard to judge it is innovativeness, which is decided by the objective of scientific researches. Innovativeness can not only represent in the new fields which predecessors have not set foot in, the questions which predecessors have not studied or solved, but also represent in the new views which predecessors have not put forward(1). By consulting and tidying up the references, the undergraduate should know all these and recognize deeply their own responsibilities gradually.

Affected by traditional educational ideas, in China the advisors could usually not exert the subjective role of the undergraduate unconsciously,requiring undergraduates to design and study their graduation theses according to the advisors' own thinking by listing subjects of graduation theses beforehand(1) Here the undergraduates have only passive options(3). Thereby thoughts of the undergraduates were usually restricted in some frames, with their independent spirits and spirit of innovation being lacked and innovative enthusiasm being inhibitted(1). This method can confound subject roles of the undergraduates easily, not conducive to arouse the problem consciousness consciousness of the undergraduates, not conducive to put in order and to rethink profoundly the basic knowledges and 
theories which they once learnt(3). It is obvious that problem consciousness is the base of innovative consciousness and innovative capability.

In addition, the advisor should foster the undergraduate's interest. Without doubt, as the undergraduates consult and tidy up the references deeply and find an unknown question which they prefer to study, their interests often increase. In fact, the course to seek fora proper thesis is also the one for advisors to communicate with undergraduates and to find their advantages and disadvantages . This is the base for advisors to guide undergraduates effectly(4).

Consolidating the undergraduates' interests with their researching theses will be the fundamental power to arouse maximumly the enthusiasm of undergraduates to do the researches and to prompt them to finish their graduation theses.

Otherwise, the graduation thesis should not only be an unresolved question, but also be under the conditions provided or provided by coordination. This is also an important standard to judge a proper thesis. It was suggested(4) that the graduation thesis decided by the advisor at last should not include so many contents that the undergraduates could not finish it on schedule. It was thought that there were some problmes such as graduation thesis selected in large range and in rare depth in agricultural university (5).

It is emphasized here that a proper thesis will be helpful to build the undergraduates' self-confidence to finish their graduation theses and cultivate their golden quality to be practical and realistic.

\section{Protocoling experiments and designing experimental methods scientifically}

Protocoling experiments and designing experimental methods will play a key role after determining the proper thesis.

An undergraduate should be guided to protocol the experiments by analyzing the characters of the question to be study, and to use directly or to improve their experimental methods by analyzing the rationality the experimental methods founded by predecessors.

Undergraduates should learn how to decide the experimental techniques and experimental methods according to the unknown problem to be explored.

For example, Wei Zhang et al (6)once added some experimental contents in the graduation thesis of geographic science and they thought that it was helpful for developing student's ability to analyze and solve problems by analyzing and concluding the data obtained from field observation and indoor experiments and that it helps meanwhile to avoid some frequent problems such as the lack of contents and innovation.

It is thought here this is an illuminating example for undergraduates to learn how to use new experimental methods to explore new knowledges to finish their graduation theses.

\section{Preparing experiments fully and doing experiments accurately}

Preparing experiments fully and doing experiments accurately will be a bridge to the results with experiments being protocoled and experimental methods being designed.

Experimental instruments and reagents are essential material conditions for experiments to be done successfully. Experiments will be out of the question without them. An undergraduate should be guided to test to ensure whether the experimental instruments can work or not, to inspect to ensure whether the experimental reagents are complete or not, to know whether the experimental materials are easy to get and to conserve or not. Undergraduates should be guided to know how they can supplement or get the things mentioned above when these things are lack.

Doing experiments accurately can be thought as the most pivotal link during all the graduation thesis stage.The graduation thesis can not be illustrated without accurate experimental results. 
Before starting formally their experiments, undergraduates should be guided to get an adaptive training, for half a month at least according to their situation, to have an intimate knowledge of the lab surroundings and experimental instruments and reagents. It is proper for them to learn and use expertly the experimental instruments and to know well every step in experimental methods, in order to get results of good repeatability. This adaptive training can also be helpful to build the undergraduates' self-confidence, which can be the spiritual power for undergraduates to overcome the difficulties to finish their graduation theses.

Once the undergraduates' experiments are put into performance from the beginning, advisors must master the schedule of experiments whenever necessary, know the problems met in the experiments and guide them to seek for the solutions, so that the experiments can be finished on schedule by guaranteeing both quality and quantity.

\section{Summarizing scientifically the experimental results, composing conscientiously the graduation thesis}

Composing the graduation thesis can not only lead to the result form of the graduation thesis, but also be a progress for the undergraduates to study to improve themselves.

It is thought here that undergraduates should learn to analyze and explain the experimental results, reveal the intrinsic connections among the researchful objects, answer accurately the theses selected properly and forecast the subsequent researches. Here revealling the intrinsic connections and forecasting the subsequent researches are the most important. All these will be useful for the undergraduates to improve their comprehensive analysis ability, to broaden their horizons and to improve their scopes and profundities to consider the questions and deal with the problems.

It is obvious that the significance of an excellent graduation thesis far exceed the score got by the undergraduate!

\section{Discussion}

Lacking innovativeness in graduation theses has been worried and come into notice in China ${ }^{(1-9)}$.

A lot of explorations have been made and some experiences have been got gradually. For example, Huayue $\mathrm{Zhu}^{(7)}$ introudced a research tutorial system which has been adopted in Taizhou University in Zhejiang in China. According to the research tutorial system, with advisors' researches being a link , the undergraduates will be directed to foster their scientific research consciousnesses and innovative abilities effectively by undertaking and fulfilling appropriate scientific research tasks. Na Wang et al ${ }^{(8)}$ introudced a project-based teaching method during graduation thesis which has been adopted in Yunnan Agricultural University in China.But its mechanism was similar to Huayue Zhu's in front.

Once in College of Architectural Science and Engineering in Yangzhou University, undergraduates of civil engineering specialty were directed to carry out their graduation theses by simulating production projects ${ }^{(9)}$. Here Peng Xiao et al thought this kind of training model caused some disadvantages. For example,the undergraduates often did not think highly of their graduation theses when thinking the simulated production projects were false. For example,the undergraduates needed not be responsible for results of the projects, for the simulated production projects could not be examined by practices, with the quality of the projects being lowered imperceptibly.For example,the undergraduates could not have chances to acquire capacity to solve practical problems in real projects. Therefor it was suggested by them that graduation theses should be decided by taking the teachers' scientific research subjects as the background and should be combined with the true scientific research subjects, with the achievements of the graduation theses being able to be applied directly to production practices .Shoujun Guo et al ${ }^{(10)}$ introduced that the graduation theses of their undergraduates showed 
two characteristics. One was that the graduation theses were selected according to the practicability reflecting the urgent problems in the society, which was useful for undergraduates to combine their professional knowledge with market demands. And the other one was that the graduation theses were selected as a part of scientific research projects of teachers showing the characteristics of scientific researches, which could reflect new standards and trends in professional development and could contribute to deepening of scientific research work.

Obviously, they all agreed above that the undergraduates should get trainings through a realistic research progress or an experimental progress. However, no matter which mode they used, the realistic research progress, an experimental progress or a scientific research project was only a practical chance or practical arena for undergraduates. As for whether undergraduates can be trained to be a serviceable talented person, the guiding role of teachers can not be ignored at all events, although this point has been mentioned in all the papers above.In fact, even if the teachers had not any projects funded by the government or companies, they could all directed the graduation theses by putting forward topics. In fact , no matter whether the name "research tutorial system" was used or not previously, the working contents mentioned in "research tutorial system" have almost long been carried out in many labs, I think.

The graduation thesis will be helpful to culture and develop students' innovative personality, innovative thinking and innovative skills ${ }^{(1)}$ and to motivate students' innovative interests and innovativeenthusiasm ${ }^{(10)}$.Undergraduates should be cultured to get abilities to find problems, to analyze problems and to solve problems, with their knowledges and abilities being practiced entirely and specialty qualities being increased ${ }^{(4)}$.

Recently a different viewpoint ${ }^{(11)}$ has arised. It suggests that graduation thesis should be canceled. Such a kind of fallacy will be discussed deeply in another paper.

Statement; This paper was supported by College of Life Science in South China Agricultural University.

\section{References}

[1] Liying Ma, Lina Li, Cultivation of Students' Innovating Ability in the Link of Writing the Graduation Thesis, Value Engineering, (2)(2009)33-35;

[2] Zhenqian Zhang, Gang Xiao, Zhenxie Yi, Chunyun Guan, Review on Quality Improvement of Undergraduates' Dissertations of Agriculture, Journal of Anhui Agri. Sci. , 42(19)(2014)6478-6480;

[3]Guoling Zhao, Si Ha, A Discussion of How to Select Topics for the Undergraduate Graduation Thesis, Journal of Inner Mongolia Finance and Economics College, 5(2)(2007)45-46;

[4] Yijun Zhou, Jinchao Feng, Likun Wang, Yingnan Zhao, The Reform in Undergraduate Teaching -Training of Diathesis in Graduate-thesis, Journal of the CUN(Natural Sciences Edition), 13(4)(2004)376-379;

[5] Haiping Li, Lingzh Li, Consideration on Topic Selection for Undergraduate Thesis in Agricultural University, Journal of Hebei Agricultural Sciences, 14 (1)(2010)158-159, 162;

[6] Wei Zhang, Lili Zhang, Analysis on the choice of experiment subjects in graduation thesis and the improvement of the thesis, Journal of Guizhou Normal College, 30(6)

(2014)31-34;

[7] Huayue Zhu, Research and Practice of Guidance Mode of Graduation Theses based on Research Tutorial System, Guangdong Chemical Industry, 41(11)(2014)259-260; 
[8] Na Wang, Guomin $\mathrm{Cu}$, Wei Huang, Gaokun Zhao, Zhengxiong Zhao, Ye Yang,A study of the effects of project-based teaching method on graduation thesis teaching for tobacco-major students, Journal of Yunnan Agricultural University, 8(4)(2014)51-54;

[9] Peng Xiao, Aihong Kang, Zhengguang Wu, Yongmei Guo, Xinglong Zhu, Graduation thesis combining with scientific research subject innovative practice promoting applied production, Journal of Architectural Education in Insitutions of Higher Learning, 14(3)(2005)69-71;

[10] Shoujun Guo, Yongli Yang, Ji Zhang, Minyun Zhao, Jian Yao, Ailun Huang, Strengthen the instruction of thesis, cultivate innovation abilities of college students, Journal of Northwest Normal University (Natural Science),40(2)(2004)103-105;

[11] Jiahui Rao, Naisheng Zhang, Analysis of comprehensive universities attempt to cancel undergraduate thesis, Journal of Higher Education Management, 8(3)(2014)

100-104; 